\title{
On a Conjecture regarding Fisher Information
}

\author{
Angelo Plastino, ${ }^{1}$ Guido Bellomo, ${ }^{1}$ and Angel Ricardo Plastino ${ }^{2}$ \\ ${ }^{1}$ Instituto de Física La Plata (IFLP-CONICET) and Departamento de Física, Facultad de Ciencias Exactas, \\ Universidad Nacional de La Plata, No. 115 and No. 49, C.C. 67, 1900 La Plata, Argentina \\ ${ }^{2}$ CeBio y Secretaria de Investigacion, Universidad Nacional del Noroeste de la Provincia de Buenos Aires-UNNOBA and CONICET, \\ Roque Saenz Pena 456, Junin, Argentina
}

Correspondence should be addressed to Guido Bellomo; gbellomo@fisica.unlp.edu.ar

Received 10 December 2014; Revised 23 January 2015; Accepted 23 January 2015

Academic Editor: Xiao-Jun Yang

Copyright (C) 2015 Angelo Plastino et al. This is an open access article distributed under the Creative Commons Attribution License, which permits unrestricted use, distribution, and reproduction in any medium, provided the original work is properly cited.

Fisher's information measure $I$ plays a very important role in diverse areas of theoretical physics. The associated measures $I_{x}$ and $I_{p}$, as functionals of quantum probability distributions defined in, respectively, coordinate and momentum spaces, are the protagonists of our present considerations. The product $I_{x} I_{p}$ has been conjectured to exhibit a nontrivial lower bound in Hall (2000). More explicitly, this conjecture says that for any pure state of a particle in one dimension $I_{x} I_{p} \geq 4$. We show here that such is not the case. This is illustrated, in particular, for pure states that are solutions to the free-particle Schrödinger equation. In fact, we construct a family of counterexamples to the conjecture, corresponding to time-dependent solutions of the free-particle Schrödinger equation. We also conjecture that any normalizable time-dependent solution of this equation verifies $I_{x} I_{p} \rightarrow 0$ for $t \rightarrow \infty$.

\section{Introduction}

A very important information measure, with manifold physical applications, was conceived by R. A. Fisher in the 1920 sfor detailed discussions see [1-4]. Recent developments show that Fisher's information has a fundamental role in quantum mechanics [5-19]. In particular, it allows for the formulation of new quantum uncertainty principles [20-24]. It is usually abbreviated as $I$ and can be thought of as a measure of the expected error in a measurement [1]

A particular instance of great relevance is that of translational families [1]. These are distribution functions whose form remains invariant under displacements of a shift parameter $\theta$. Thus, they are shift invariant distributions (in a Mach sense, there is no absolute origin for $\theta$ ). The measure exhibits Galilean invariance [1]. Given a probability density $f(\mathbf{x}, \theta)$, with $\mathbf{x} \in \mathbb{R}^{D}$ and $\theta=\left(\theta_{i}\right)_{1<i<n}$ a family of parameters, the concomitant Fisher matrix is [25]

$$
I_{j k}:=\int \frac{1}{f(\mathbf{x}, \theta)}\left(\frac{\partial f}{\partial \theta_{j}}\right)\left(\frac{\partial f}{\partial \theta_{k}}\right) \mathrm{d} \mathbf{x}
$$

where $\mathrm{d} \mathbf{x}=\prod_{k=1}^{D} \mathrm{~d} x_{k}$ is the volume element in $\mathbb{R}^{D}$. In particular, for $\theta \in \mathbb{R}^{D}$, one defines translational families $f(\mathbf{x}-\theta)$, with elements $I_{j k}=\int(1 / f)\left(\partial_{j} f\right)\left(\partial_{k} f\right) \mathrm{d} \mathbf{x}$, where $\partial_{i}$ represents the partial derivative with respect to the coordinate $x_{i}$. The trace of this matrix, given by $I=$ $\int(1 / f)\left[\sum_{k=1}^{D}\left(\partial_{k} f\right)^{2}\right] \mathrm{d} \mathbf{x}$, is a good uncertainty indicator for probability distributions associated with quantum wave functions [26]. If $\psi(\mathbf{x})$ is a normalized wave function in coordinate space $\left(D\right.$-dimensions) and $\widetilde{\psi}(\mathbf{p})=(2 \pi)^{-D / 2} \int e^{-\mathbf{i x} \cdot \mathbf{p}} \psi(\mathbf{x}) \mathrm{d} \mathbf{x}$ is its momentum-counterpart, the corresponding probability densities are, respectively, $\rho(\mathbf{x})=|\psi(\mathbf{x})|^{2}$ and $\widetilde{\rho}(\mathbf{p})=|\widetilde{\psi}(\mathbf{p})|^{2}$, with associated Fisher measures

$$
\begin{aligned}
& I_{\mathbf{x}}=\int \frac{1}{\rho}\left[\nabla_{\mathbf{x}} \rho\right]^{2} \mathrm{~d} \mathbf{x}, \\
& I_{\mathbf{p}}=\int \frac{1}{\widetilde{\rho}}\left[\nabla_{\mathbf{p}} \tilde{\rho}\right]^{2} \mathrm{~d} \mathbf{p},
\end{aligned}
$$

which allow one to study uncertainty relations via the product $I_{\mathrm{x}} I_{\mathrm{p}}[26]$. 
For instance, one can demonstrate that if $\psi(\mathbf{x})$ (or $\widetilde{\psi}(\mathbf{p}))$ is real, then $I_{\mathbf{x}} I_{\mathbf{p}} \geq 4 D^{2}$ [5], with equality for coherent states of the harmonic oscillator (HO) [26]. For general, mixed states it is clear that the product $I_{\mathbf{x}} I_{\mathrm{p}}$ does not possess a nontrivial lower bound (e.g., one can use thermal HO states, represented by Gaussian distributions in both coordinates and momenta, in the high temperature limit). In the case of pure states, though, the existence of such a lower bound for $I_{\mathbf{x}} I_{\mathbf{p}}$ was an open question. Hall conjectured that the relation $I_{x} I_{p} \geq 4$ might hold in general for pure states in one dimension [26, page 3]. We will next present a couple of counterexamples that show this conjecture to be incorrect. Our examples give rise to a new conjecture: for a bounded wave function one has $I_{\mathbf{x}} I_{\mathbf{p}} \rightarrow 0$ when $t \rightarrow \infty$.

\section{Counterexamples}

Our first example is taken from the considerations (in a different context) of [5]. A free-particle's one-dimensional wave packet $\psi(x, t)$ (unit mass) evolves according to Schrödinger's equation

$$
\mathbf{i} \frac{\partial \psi}{\partial t}=-\frac{1}{2} \frac{\partial^{2} \psi}{\partial x^{2}} .
$$

Setting the initial conditions

$$
\begin{gathered}
\psi(x, 0)=A_{0} \exp \left[-\frac{x^{2}}{2 \Delta^{2}}\right], \\
\widetilde{\psi}(p, 0)=\widetilde{A}_{0} \exp \left[-\frac{\Delta^{2} p^{2}}{2}\right],
\end{gathered}
$$

with $A_{0}=\Delta^{-1 / 2} \pi^{-1 / 4}, \widetilde{A}_{0}=\Delta^{1 / 2} \pi^{-1 / 4}$, and $\Delta>0$, that correspond to a Gaussian packet, one finds the solution

$$
\psi(x, t)=A(t) \exp \left[-\frac{x^{2}}{2 \Delta^{2}\left(1+\mathbf{i} t / \Delta^{2}\right)}\right],
$$

where $A(t)=A_{0}\left(1+\mathbf{i} t / \Delta^{2}\right)^{-1 / 2}$. The associated probability densities are

$$
\begin{gathered}
\rho(x, t)=\frac{\Delta}{\sqrt{\pi\left(\Delta^{4}+t^{2}\right)}} \exp \left[-\frac{\Delta^{2} x^{2}}{\Delta^{4}+t^{2}}\right], \\
\tilde{\rho}(p, t)=\frac{\Delta}{\sqrt{\pi}} \exp \left[-\Delta^{2} p^{2}\right] .
\end{gathered}
$$

The product $I_{x} I_{p}=4 \Delta^{4}\left(\Delta^{4}+t^{2}\right)^{-1}$ obeys the relation $I_{x} I_{p}<4$ for $t>0$. Also, one has $I_{x} I_{p} \rightarrow 0$ for $t \rightarrow \infty$.

We pass now to another free-particle solution, given by the first partial derivative of $\psi(x, t)$ with respect to $x$ : $\psi^{(1)}(x, t) \propto \partial_{x} \psi(x, t)($ see $(4))$, correctly normalized. It is easy to see that $\psi^{(1)}(x, t)$ is a solution by deriving both members of (4); that is,

$$
\mathbf{i} \frac{\partial}{\partial t} \frac{\partial \psi}{\partial x}=-\frac{1}{2} \frac{\partial^{2}}{\partial x^{2}} \frac{\partial \psi}{\partial x}
$$

The new solution is

$$
\psi^{(1)}(x, t)=A^{(1)}(t) \exp \left[-\frac{x^{2}}{2 \Delta^{2}\left(1+\mathbf{i} t / \Delta^{2}\right)}\right],
$$

with $A^{(1)}(t)=-2^{1 / 2} \pi^{1 / 4} \Delta^{3 / 2}\left(\Delta^{2}+\mathbf{i} t\right)^{-3 / 2}$. The two corresponding densities are

$$
\begin{gathered}
\rho^{(1)}(x, t)=\frac{2 \Delta^{3}}{\sqrt{\pi\left(\Delta^{4}+t^{2}\right)^{3}}} x^{2} \exp \left[-\frac{\Delta^{2} x^{2}}{\Delta^{4}+t^{2}}\right], \\
\widetilde{\rho}^{(1)}(p, t)=\frac{2 \Delta^{3}}{\sqrt{\pi}} p^{2} \exp \left[-\Delta^{2} p^{2}\right] .
\end{gathered}
$$

The product is $I_{x}^{(1)} I_{p}^{(1)}=36 \Delta^{4}\left(\Delta^{4}+t^{2}\right)^{-1}$, verifying $I_{x}^{(1)} I_{p}^{(1)}<4$ for $t>2 \sqrt{2} \Delta^{2}$, and $I_{x}^{(1)} I_{p}^{(1)} \rightarrow 0$ when $t \rightarrow \infty$.

In general, one can show that the whole family of solutions of (4) given by successive derivatives of $\psi(x, t)$, that is, the set $\left\{\psi^{(n)}(x, t) \mid \psi^{(n)}(x, t)=N_{n} \partial_{x}^{n} \psi(x, t), n=0,1,2, \ldots\right\}$, verifies that $I_{x} I_{p} \rightarrow 0$ when $t \rightarrow \infty$, with $N_{n}$ the pertinent normalization constants. Thus, the family $\left\{\psi^{(n)}(x, t)\right\}_{n \in \mathbb{N}_{0}}$ yields infinite counterexamples to Hall's conjecture. To see this, one needs first to rewrite the Fisher measure in wave function's terms, so that (2) becomes equivalent to

$$
I_{\mathbf{x}}=4 \int\left(\nabla_{\mathbf{x}}|\psi|\right)^{2} \mathrm{~d} \mathbf{x},
$$

or, in one dimension, $I_{x}=\int\left(\partial_{x}|\psi|\right)^{2} \mathrm{~d} x$. Further, $|\psi|=\psi^{*} \psi$. Thus, $I_{x}$ can be expressed in terms of $\psi$ and $\psi^{(1)}$. In one dimension one has

$$
I_{x}=4 \int\left(\psi^{(1) *} \psi+\psi^{*} \psi^{(1)}\right)^{2} \mathrm{~d} x .
$$

In general, for $\psi^{(k)}$, the Fisher's measure associated with the distribution $\left|\psi^{(k)}\right|^{2}$ becomes

$$
I_{x}^{(k)}=4 \int\left(\psi^{(k+1) *} \psi^{(k)}+\psi^{(k) *} \psi^{(k+1)}\right)^{2} \mathrm{~d} x .
$$

We show now that the integrand tends to zero for $t \rightarrow \infty$. Thus, $I_{x}^{(k)} \rightarrow 0$ in such a limit. Actually, we will show that $\psi^{(k)}(x, t) \rightarrow 0$ for $t \rightarrow \infty$. The $k$ th derivative of $\psi(x, t) \equiv$ $\psi^{(0)}(x, t)$ is proportional to the $k$ th derivative of a Gaussian distribution, given by

$$
\begin{aligned}
\psi^{(k)}(x, t) & =N_{k}(t) \frac{\partial^{k}}{\partial x^{k}} \psi^{(0)}(x, t) \\
& =N_{k}(t) \frac{\partial^{k}}{\partial x^{k}}\left(A(t) e^{-c(t)^{2} x^{2}}\right) \\
& =N_{k}(t) A(t)(-1)^{k} c(t)^{2} H_{k}(c(t) x) e^{-c(t)^{2} x^{2}} \\
& =N_{k}(t)(-1)^{k} c(t)^{2} H_{k}(c(t) x) \psi^{(0)}(x, t),
\end{aligned}
$$

where $c(t)^{2}=\left(2\left(\Delta^{2}+\mathbf{i} t\right)\right)^{-1}$ and $H_{k}(y)$ is the Hermite polynomial of degree $k$ in the variable $y$. The time-dependent 
parameters $c(t), \psi^{(0)}(x, t)$, and $A(t)$ vanish for $t \rightarrow \infty$. What is the behavior of $N_{k}$ ? Let us see what happens with $\widetilde{\psi}^{(k)}(p, t)$, the $k$ th solution in momentum space, corresponding to the Fourier transform of $\psi^{(k)}(x, t)$. We have

$$
\begin{aligned}
\tilde{\psi}^{(k)}(p, t) & =\frac{1}{\sqrt{2 \pi}} \int e^{-\mathbf{i} x p} \psi^{(k)}(x, t) \mathrm{d} x \\
& =N_{k}(t) A(t) \int e^{-\mathbf{i} x p} \frac{\partial^{k}}{\partial x^{k}} e^{-c(t)^{2} x^{2}} \mathrm{~d} x \\
& =N_{k}(t) A(t)(\mathbf{i} p)^{k} \frac{1}{\sqrt{2} c(t)} \exp \left[-\frac{p^{2}}{4 c(t)^{2}}\right] \\
& =N_{k}(t) \sqrt{c(t)}(\mathbf{i} p)^{k} \widetilde{\psi}^{(0)}(p, t) .
\end{aligned}
$$

Demanding normalization leads to

$$
\begin{aligned}
1 & =\int \widetilde{\psi}^{(k)}(p, t) \widetilde{\psi}^{(k) *}(p, t) \mathrm{d} p \\
& =\left|N_{k}(t)\right|^{2}|c(t)| \int p^{2 k}\left|\widetilde{\psi}^{(0)}\right|^{2} \mathrm{~d} p \\
& =\left|N_{k}(t)\right|^{2}|c(t)| \frac{\Delta}{\sqrt{\pi}} \int p^{2 k} e^{-\Delta^{2} p^{2}} \mathrm{~d} p \\
& =\left|N_{k}(t)\right|^{2}|c(t)| \frac{\Delta}{\sqrt{\pi}} \Gamma\left(k+\frac{1}{2}\right) \Delta^{-2 k-1} .
\end{aligned}
$$

Thus,

$$
\left|N_{k}(t)\right|^{2}=\frac{\sqrt{\pi} \Delta^{2 k}}{\Gamma(k+1 / 2)} 2 \sqrt{\Delta^{4}+t^{2}} .
$$

Remember that

$$
\psi^{(0)}(x, t)=A(t) e^{-c(t)^{2} x^{2}},
$$

with $A(t)=\pi^{-1 / 4} \sqrt{2 \Delta} c(t)$, and

$$
\widetilde{\psi}^{(0)}(p, t)=\frac{\sqrt{\Delta}}{\pi^{1 / 4}} \exp \left[-\frac{p^{2}}{4 c(t)^{2}}\right] .
$$

One finds the following limits for the absolute values of the wave functions:

$$
\begin{aligned}
\left|\psi^{(k)}(x, t)\right|^{2} & =\left|N_{k}(t) c(t)^{2} H_{k}(c(t) x) \psi^{(0)}(x, t)\right|^{2} \\
& \sim|c(t)|^{-1}|c(t)|^{4}|c(t)|^{2 k}|c(t)|^{2} e^{-2 \Re\left(c(t)^{2}\right) x^{2}} \\
& \sim|c(t)|^{2 k+5} \exp \left[-\frac{\Delta^{2} x^{2}}{\Delta^{4}+t^{2}}\right] \underset{t \rightarrow \infty}{\longrightarrow} 0 \\
\left|\widetilde{\psi}^{(k)}(p, t)\right|^{2} & =\left|N_{k}(t) \sqrt{c(t)}(\mathbf{i} p)^{k} \widetilde{\psi}^{(0)}(p, t)\right|^{2} \\
& \sim|c(t)|^{-1}|c(t)|\left|\exp \left[-\frac{p^{2}}{4 c(t)^{2}}\right]\right|^{2} \\
& =e^{-\Delta^{2} p^{2}}
\end{aligned}
$$

Equation (20) indicates that all functions $\psi^{(k)}(x, t)$ vanish for $t \rightarrow \infty$. Accordingly, from (13) we find $I_{x}^{(k)} \rightarrow 0$ for $t \rightarrow \infty$ for all $k=0,1,2, \ldots$. Further, $(21)$ shows that $\widetilde{\psi}^{(k)}(p, t)$ does not vanish in this limit. In fact, $\left|\widetilde{\psi}^{(k)}(p, t)\right|$ does not depend on $t$.

So as to understand what happens with $I_{p}^{(k)}$ let us see an expression analogous to (13) in momentum space:

$$
I_{p}^{(k)}=4 \int\left(\widetilde{\psi}^{(k+1) *} \tilde{\psi}^{(k)}+\widetilde{\psi}^{(k) *} \widetilde{\psi}^{(k+1)}\right)^{2} \mathrm{~d} x .
$$

Expanding the integrand using (15) we have

$$
\begin{aligned}
& \widetilde{\psi}^{(k+1) *} \widetilde{\psi}^{(k)}+\widetilde{\psi}^{(k) *} \widetilde{\psi}^{(k+1)} \\
& \quad=2 \mathfrak{J}\left(N_{k} N_{k+1}^{*}\right)|c(t)| p^{2 k+1}\left|\widetilde{\psi}^{(0)}(p, t)\right|^{2} .
\end{aligned}
$$

Introducing this into (22), and remembering that both $N_{k}$ and $c(t)$ are independent of $p$, we find

$$
\begin{aligned}
I_{p}^{(k)} & =16 \Im\left(N_{k} N_{k+1}^{*}\right)^{2}|c(t)|^{2} \frac{\Delta^{2}}{\pi} \int p^{4 k+2} e^{-2 \Delta^{2} p^{2}} \\
& =16 \Im\left(N_{k} N_{k+1}^{*}\right)^{2}|c(t)|^{2} \frac{\Delta^{2}}{\pi} \frac{\Gamma(2 k+3 / 2)}{\left(2 \Delta^{2}\right)^{2 k+3 / 2}} .
\end{aligned}
$$

Since $\left|N_{k}(t)\right|^{2} \sim|c(t)|^{-1}$ (see (16)), one has $\left|N_{k}(t) N_{k+1}\right|^{2}|c(t)|^{2} \sim 1$ and thus $I_{p}^{(k)}$ becomes bounded. Thus, there exists $I_{p \text {,max }}^{(k)} \in \mathbb{R}_{>0}$ such that $I_{p}^{(k)} \leq I_{p \text {,max }}^{(k)}$ for all $k=0,1,2, \ldots$. We conclude that $I_{x}^{(k)} I_{p}^{(k)} \rightarrow 0$ for $t \rightarrow \infty$ for the whole family of solutions $\left\{\psi^{(k)}(x, t)\right\}_{k \in \mathbb{N}_{0}}$.

\section{Conclusions}

We conclude by reiterating that we have found an infinite number of counterexamples to the conjecture $I_{x} I_{p} \geq 4$, for pure states, put forward in [26]. On the basis of these results, we conjecture that, for any normalizable wave function $\psi(x, 0)$, the corresponding time-dependent solution $\psi(x, t)$ of the free-particle Schrödinger equation satisfies $I_{x} I_{p} \rightarrow 0$ for $t \rightarrow \infty$.

\section{Conflict of Interests}

The authors declare that there is no conflict of interests regarding the publication of this paper.

\section{References}

[1] B. R. Frieden, Physics from Fisher Information, Cambridge University Press, Cambridge, UK, 1998.

[2] B. R. Frieden, Science from Fisher Information, Cambridge University Press, Cambridge, UK, 2004.

[3] R. Carroll, On the Emergence Theme of Physics, World Scientific, Singapore, 2010.

[4] F. Pennini and A. Plastino, "Heisenberg-Fisher thermal uncertainty measure," Physical Review E, vol. 69, no. 5, Article ID 057101, 2004. 
[5] P. Sánchez-Moreno, A. R. Plastino, and J. S. Dehesa, "A quantum uncertainty relation based on Fisher's information," Journal of Physics. A. Mathematical and Theoretical, vol. 44, no. 6, Article ID 065301, 2011.

[6] I. V. Toranzo and J. S. Dehesa, "Entropy and complexity properties of the d-dimensional blackbody radiation," The European Physical Journal D, vol. 68, article 316, 2014.

[7] J. S. Dehesa, A. R. Plastino, P. Sánchez-Moreno, and C. Vignat, "Generalized Cramér-Rao relations for non-relativistic quantum systems," Applied Mathematics Letters, vol. 25, no. 11, pp. 1689-1694, 2012.

[8] J. S. Dehesa, R. O. Esquivel, A. R. Plastino, and P. SanchezMoreno, "Fisher information: uncertainty relation and steric effect," Journal of Russian Laser Research, vol. 32, no. 5, pp. 403411, 2011.

[9] B. R. Frieden, A. Plastino, A. R. Plastino, and B. H. Soffer, "Schrödinger link between nonequilibrium thermodynamics and Fisher information," Physical Review E: Statistical, Nonlinear, and Soft Matter Physics, vol. 66, no. 4, Article ID 046128, 2002.

[10] A. Puente, A. R. Plastino, M. Casas, F. Garcias, and A. Plastino, "Fisher information and quantum systems with positiondependent effective mass," Physica A: Statistical Mechanics and its Applications, vol. 277, no. 1, pp. 146-156, 2000.

[11] M. Reginatto, "Erratum: derivation of the equations of nonrelativistic quantum mechanics using the principle of minimum Fisher information [Phys. Rev. A 58, 1775 (1998)]," Physical Review A, vol. 60, no. 2, p. 1730, 1999.

[12] Á. Nagy and E. Romera, "Relation between Fisher measures of information coming from pair distribution functions," Chemical Physics Letters, vol. 490, pp. 242-244, 2010.

[13] K. Sen, J. Antolin, and J. Angulo, "Fisher-Shannon analysis of ionization processes and isoelectronic series," Physical Review A, vol. 76, no. 3, Article ID 032502, 7 pages, 2007.

[14] S. Luo, "Fisher information, kinetic energy and uncertainty relation inequalities," Journal of Physics A: Mathematical and General, vol. 35, no. 25, pp. 5181-5187, 2002.

[15] P. Facchi, R. Kulkarni, V. I. Man'ko, G. Marmo, E. C. Sudarshan, and F. Ventriglia, "Classical and quantum Fisher information in the geometrical formulation of quantum mechanics," Physics Letters A, vol. 374, no. 48, pp. 4801-4803, 2010.

[16] D. Girolami, T. Tufarelli, and G. Adesso, "Characterizing nonclassical correlations via local quantum uncertainty," Physical Review Letters, vol. 110, no. 24, Article ID 240402, 5 pages, 2013.

[17] J. R. Choi, M.-S. Kim, D. Kim, M. Maamache, S. Menouar, and I. H. Nahm, "Information theories for time-dependent harmonic oscillator," Annals of Physics, vol. 326, no. 6, pp. 1381-1393, 2011.

[18] J. Liu, X. X. Jing, W. Zhong, and X. Wang, "Quantum fisher information for density matrices with arbitrary ranks," Communications in Theoretical Physics, vol. 61, no. 1, pp. 45-50, 2014.

[19] S. B. Nicholson and E. J. Kim, Physics Letters A. In press.

[20] S. Luo, "Quantum fisher information and uncertainty relations," Letters in Mathematical Physics, vol. 53, no. 3, pp. 243-251, 2000.

[21] E. Romera, P. Sánchez-Moreno, and J. S. Dehesa, “The Fisher information of single-particle systems with a central potential," Chemical Physics Letters, vol. 414, no. 4-6, pp. 468-472, 2005.

[22] P. Sánchez-Moreno, R. González-Férez, and J. S. Dehesa, “ Improvement of the Heisenberg and Fisher-information-based uncertainty relations for $D$-dimensional central potentials," New Journal of Physics, vol. 8, article 330, 2006.
[23] J. S. Dehesa, R. González-Férez, and P. Sánchez-Moreno, "The Fisher-information-based uncertainty relation, CramerRao inequality and kinetic energy for the $D$-dimensional central problem," Journal of Physics A: Mathematical and Theoretical, vol. 40, no. 8, pp. 1845-1856, 2007.

[24] G. Tóth and D. Petz, "Extremal properties of the variance and the quantum Fisher information," Physical Review A, vol. 87, no. 3, Article ID 032324, 2013.

[25] B. R. Frieden and B. H. Soffer, "Lagrangians of physics and the game of Fisher-information transfer," Physical Review E, vol. 52, no. 3, pp. 2274-2286, 1995.

[26] M. J. Hall, "Quantum properties of classical Fisher information," Physical Review A, vol. 62, no. 1, 2000. 


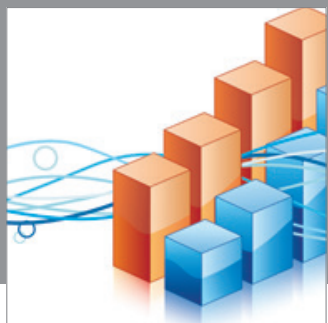

Advances in

Operations Research

mansans

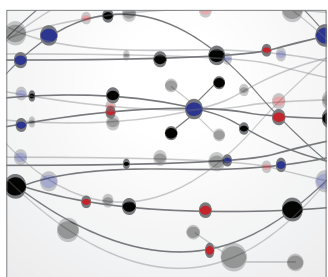

The Scientific World Journal
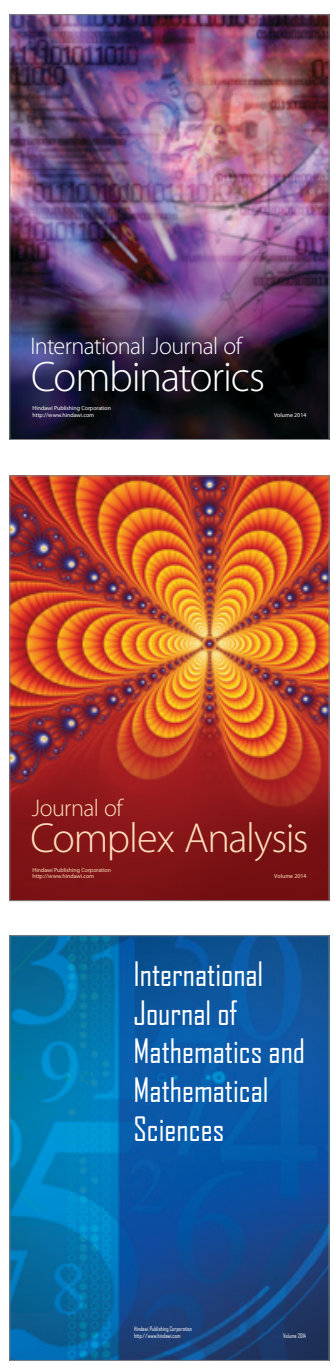
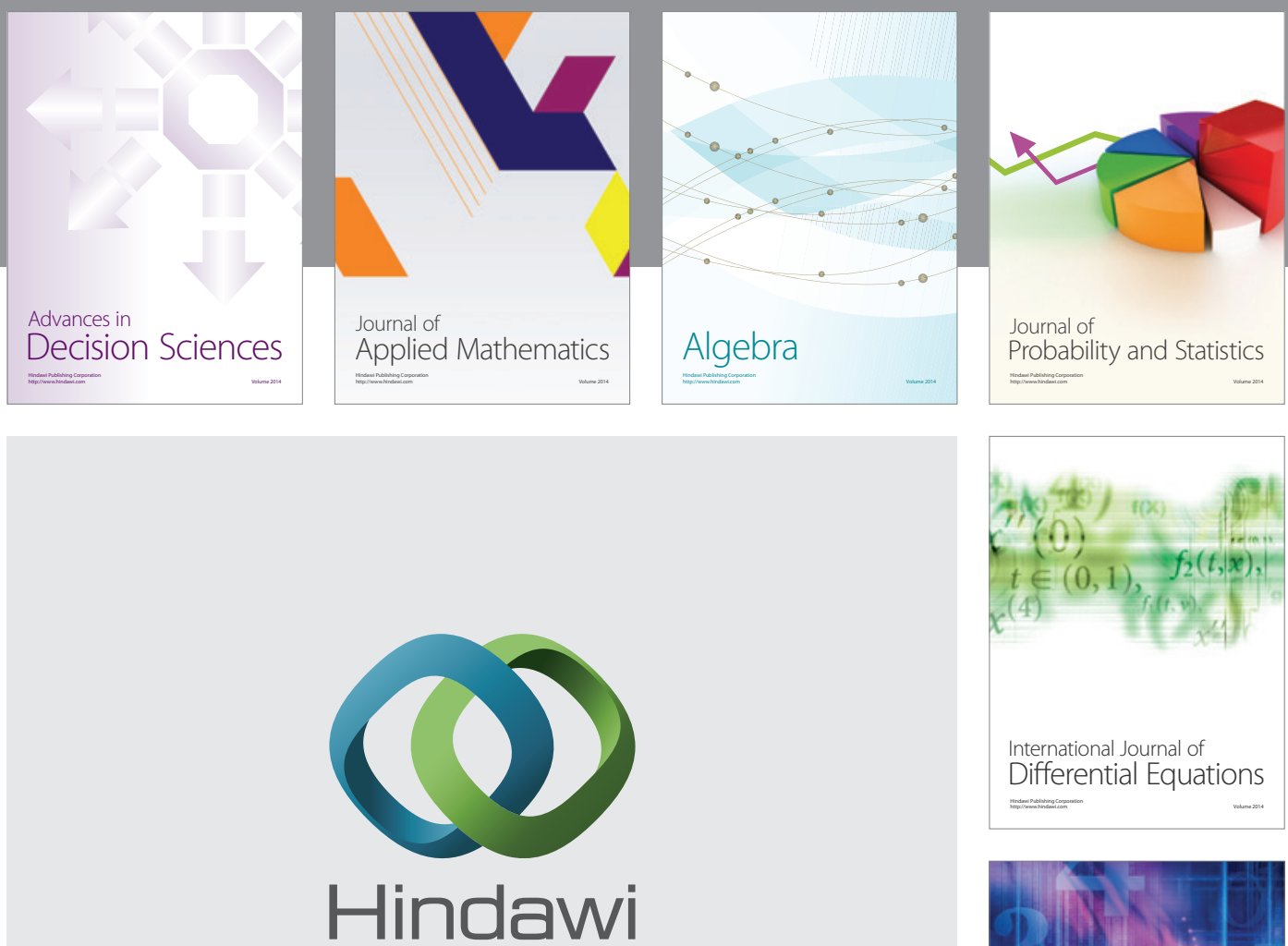

Submit your manuscripts at http://www.hindawi.com
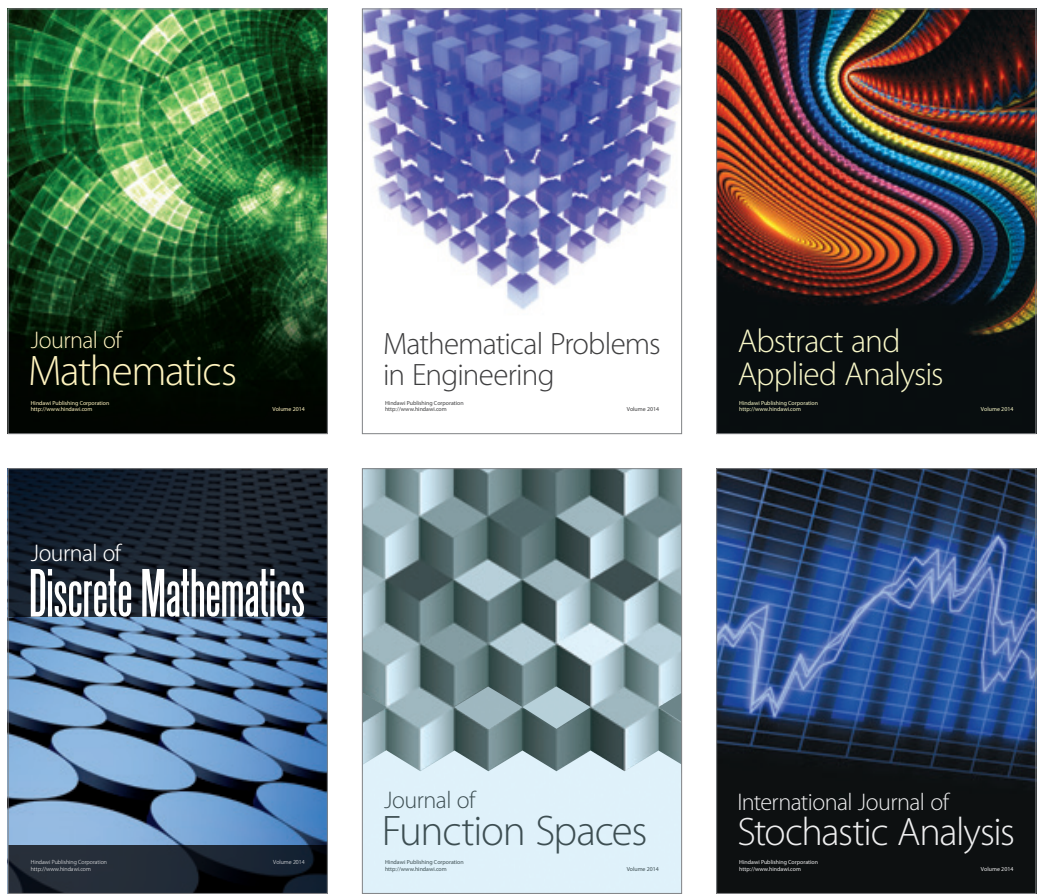

Journal of

Function Spaces

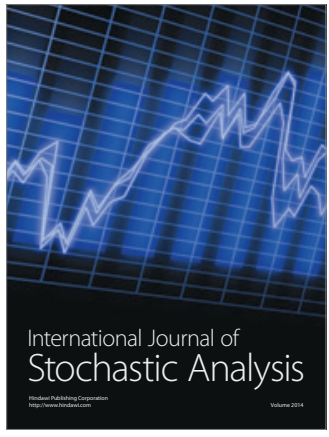

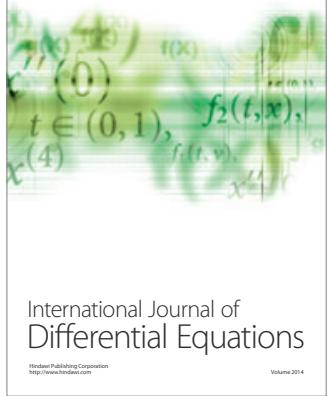
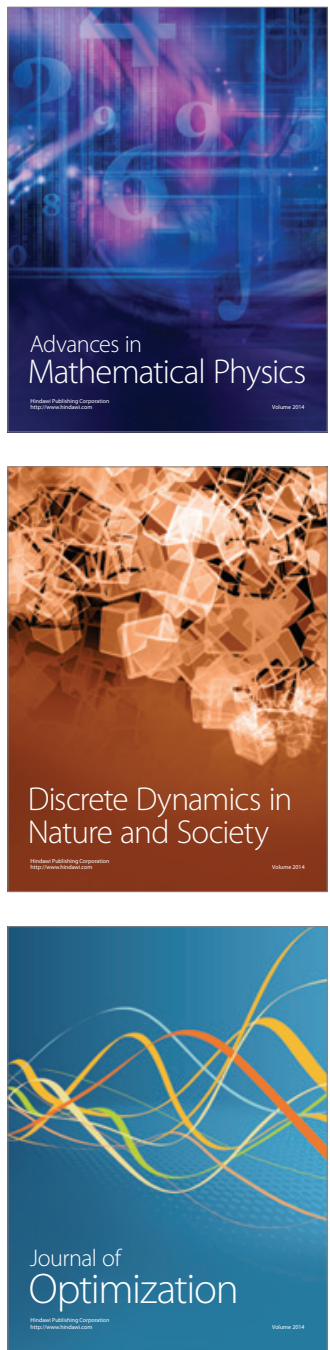